Proceedings of the 1999 Particle Accelerator Conference, New York, 1999

\title{
COHERENT SPONTANEOUS EMISSION IN HIGH GAIN FREE-ELECTRON LASERS*
}

\author{
Zhirong Huang and Kwang-Je Kim, APS/ANL, Argonne, IL 60439
}

\section{Abstract}

We investigate finite pulse effects in self-amplified spontaneous emission (SASE), especially the role of coherent spontaneous emission (CSE) in the start and the evolution of the free-electron laser (FEL) process. When the FEL interaction is negligible, we solve the one-dimensional Maxwell equation exactly and clarify the meaning of the slowly varying envelope approximation (SVEA). In the exponential gain regime, we solve the coupled VlasovMaxwell equations and extend the linear theory to a bunched beam with energy spread. A time-dependent, nonlinear simulation algorithm is employed to study the CSE effect for a general beam distribution.

\section{INTRODUCTION}

Coherent spontaneous emission (CSE) has attracted much attention as the electron bunches become shorter and more intense in current experiments demonstrating the principle of self-amplified spontaneous emission (SASE). The onedimensional (1D) theory of SASE $[1,2]$ is based on the solution of the linearized Vlasov-Maxwell equations, for the cases of a coasting beam with energy spread [1] and a bunched monochromatic beam [2]. Attempts have been made to include the energy spread for a bunched beam, but only coherent bunching at the resonant wavelength was considered [3]. In Ref. [4], the evolution of the electric field is studied with the individual particle formulation for a bunched monochromatic beam, and the contribution of the incoherent and the coherent SASE are identified. In this paper, we extend the linear theory to a bunched beam with energy spread and calculate the effect of CSE for the high gain FEL. We also present a time-dependent, nonlinear simulation algorithm that takes CSE into account for an arbitrary beam distribution.

\section{COHERENT SPONTANEOUS EMISSION}

The 1D Maxwell equation for the transverse electric field of a plane wave propagating along the undulator axis $z$ is

$$
\left(\frac{\partial^{2}}{\partial z^{2}}-\frac{1}{c^{2}} \frac{\partial^{2}}{\partial t^{2}}\right) \vec{E}_{\perp}(z, t)=\mu_{0} \frac{\partial}{\partial t} \vec{J}_{\perp}(z, t)
$$

where $\mu_{0}$ is the permeability of free space, and the charge density term is absent here due to transverse uniformity. Writing the transverse current as $\vec{J}_{\perp}(z, t)=\vec{e} J(z, t)+$ c.c.,

\footnotetext{
* Work supported by U.S. Department of Energy, Office of Basic Energy Sciences, under Contract No. W-31-109-ENG-38
}

with $\vec{e}=\hat{x}$ (a unit vector in the $x$ direction) for a planar undulator and $\vec{e}=\hat{x} \pm i \hat{y}$ for a helical undulator, we have

$$
J=-\frac{e c}{2 \sigma} \frac{K}{\gamma_{0}} e^{-i k_{u} z} \sum_{j=1}^{N} \delta\left(z-z_{j}(t)\right) \Theta\left(t-t_{j}\right),
$$

where $\sigma$ is the beam cross section, $K$ is the undulator strength parameter for the helical undulator and $K[J J]$ for the planar undulator after averaging over the undulator pe$\operatorname{riod} k_{u}$. We have also assumed that the $j^{t h}$ electron enters the undulator at $t=t_{j}(j=1, \ldots, N)$ and $z=0$. Thus, the longitudinal position of the electron is $z_{j}(t)=\beta c\left(t-t_{j}\right)$, where $\beta c$ is the average longitudinal velocity. $\Theta(t)$ is the step function, i.e., $\Theta(t)=1$ for $t>0$ and 0 otherwise.

In the absence of FEL interaction, the electric field in the form $\vec{E}_{\perp}=\vec{e} E(z, t)+$ c.c is found to be

$$
\begin{gathered}
E=\kappa_{1} \sum_{j=1}^{N}\left\{\frac{e^{i k_{f}\left[z-c\left(t-t_{j}\right)\right]}}{(1-\beta)}\left[\beta c\left(t-t_{j}\right)<z<c\left(t-t_{j}\right)\right]\right. \\
+\frac{e^{-i k_{b}\left[z+c\left(t-t_{j}\right)\right]}}{(1+\beta)}\left[\left(-c\left(t-t_{j}\right)<z<\beta c\left(t-t_{j}\right)\right]\right\},
\end{gathered}
$$

where $\kappa_{1}=e c Z_{0} K /\left(4 \sigma \gamma_{0}\right), Z_{0}=c \mu_{0} \approx 377 \Omega$, and the forward and the backward wavenumbers are

$$
k_{f}=\frac{k_{u} \beta}{1-\beta}, \quad k_{b}=\frac{k_{u} \beta}{1+\beta} .
$$

Equation (3) describes a sum of $N$ forward and backward wave packets, with the forward wave packets having much higher amplitude and shorter duration due to relativity.

For coherent spontaneous emission, we can define a relative position $\xi=z-\beta c t$ along the bunch and turn the sum into an integral by using the smooth approximation

$$
\sum_{j=1}^{N} \rightarrow n_{0} \int d\left(\xi^{\prime}\right) \chi\left(\xi^{\prime}\right)
$$

where $n_{0}$ is the maximum line density and $\chi(\xi)$ is the initial bunch density function $(0 \leq \chi(\xi) \leq 1)$. For a single-step pulse, $\chi(\xi)=\Theta(-\xi)$, the electric field in front of the pulse $(0<\xi<(1-\beta) z)$ is

$$
E_{c}=\frac{\kappa_{1} n_{0}}{i k_{u}}\left[1-e^{i k_{f}(z-c t)}\right] .
$$

Only the forward wave component is present as expected. The constant term terminates the field at the slippage distance $\xi_{s}=(1-\beta) z$. The electric field inside the pulse is given by

$$
E_{c}=\frac{\kappa_{1} n_{0}}{i k_{u}}\left[1-e^{-i k_{b}(z+c t)}\right]
$$


Thus, the coherent radiation comes from the sharp edge at $\xi=0$ for such a single-step pulse or from any density gradient for a general bunch distribution. A flat-top bunch can be constructed from two single-step pulses separated by the bunch length $l_{b}$, and the relative intensity of the incoherent versus the coherent spontaneous emissions can be obtained from Eqs. (3) and (6):

$$
\frac{I_{\text {incoh }}}{I_{\text {coh }}} \sim \frac{N k_{f}^{2}}{n_{0}^{2}}=\frac{\left(k_{f} l_{b}\right)^{2}}{N}=\frac{1}{N}\left(\frac{2 \pi l_{b}}{\lambda_{r}}\right)^{2},
$$

where $\lambda_{r}=(1-\beta) \lambda_{u} / \beta$ is the forward resonant wavelength. Equation (8) was derived in Ref. [5] by considering the initial coherent bunching factor. For $N \sim 10^{10}$ and $l_{b} \sim 2 \mathrm{~mm}, \lambda_{r}<120 \mathrm{~nm}$ makes the incoherent power larger, while $\lambda_{r}>120 \mathrm{~nm}$ favors the coherent power. Thus, the CSE effect should be negligible for the proposed $\mathrm{x}$-ray FEL projects, but may play a significant role in current experiments in the IR and visible region. We note that the flat-top model requires the electron density to vanish within $\lambda_{r}$ and tends to exaggerate the coherent effect.

\section{LINEAR ANALYSIS}

For FEL interaction, the backward wave is dropped and the slowly varying envelope approximation (SVEA) is invoked in the form

$$
\begin{aligned}
& E(z, t)=\mathcal{E}(z, t) e^{i k_{f}(z-c t),} \\
& J(z, t)=\mathcal{J}(z, t) e^{i k_{f}(z-c t),}
\end{aligned}
$$

where $\mathcal{E}$ and $\mathcal{J}$ are assumed to vary slowly with $z$ and $t$. The Maxwell equation becomes

$$
\left(\frac{\partial}{\partial z}+\frac{1}{c} \frac{\partial}{\partial t}\right) \mathcal{E}=-\frac{Z_{0}}{2} \mathcal{J} .
$$

It is convenient to define the electron coordinate as

$$
\theta=k_{f}(z-c t)+k_{u} z=\frac{k_{u}}{(1-\beta)}(z-\beta c t) \approx k_{f} \xi
$$

and change the independent variables from $(z, t)$ to $(z, \theta)$. From Eq. (2), the transverse current is

$$
\mathcal{J}=-\frac{e c}{2 \sigma} \frac{K}{\gamma_{0}} k_{f} \sum_{j=1}^{N} \delta\left(\theta-\theta_{j}\right) e^{-i \theta}
$$

where $\theta_{j}=-k_{f} c t_{j}$. Inserting this into Eq. (10), we reproduce the forward wave component of Eq. (3).

The phase space distribution of the electron beam is given by the Klimontovich distribution [1]

$$
F(\theta, \eta, z)=\frac{k_{f}}{n_{0}} \sum_{j=1}^{N} \delta\left(\theta-\theta_{j}(z)\right) \delta\left(\eta-\eta_{j}(z)\right),
$$

where $\eta=\left(\gamma-\gamma_{0}\right) / \gamma_{0}$ is the conjugate variable to $\theta$. Equation (10) can now be written as

$$
\left(\frac{\partial}{\partial z}+k_{u} \frac{\partial}{\partial \theta}\right) \mathcal{E}=\kappa_{1} n_{0} e^{-i \theta} \int d \eta F(\theta, \eta, z) .
$$

The Vlasov equation for the electron distribution is $[1,2]$

$$
\frac{\partial F}{\partial z}+2 k_{u} \eta \frac{\partial F}{\partial \theta}-\kappa_{2} \mathcal{E} e^{i \theta} \frac{\partial F}{\partial \eta}=0,
$$

where $\kappa_{2}=e K /\left(2 \gamma_{0}^{2} m c^{2}\right)$ is a constant.

In the exponential gain regime and without external field, we can regard the electric field in Eq. (15) as a small, firstorder quantity. This includes the coherent and the incoherent spontaneous emissions as well as the stimulated emission. Hence the distribution function $F$ consists of two terms: the zeroth-order term is the initial smooth distribution given by

$$
F_{0}(\theta, \eta, z)=\chi\left(\theta-2 k_{u} \eta z\right) V(\eta)
$$

where $V(\eta)$ is the initial energy spread of the beam normalized to $\int d \eta V(\eta)=1$, and the first-order term $\Delta F$ contains both the initial fluctuation $\Delta F_{0}$ and the bunching behavior through FEL interaction. Approximating $F$ with $F_{0}$ in the third term of Eq. (15) yields

$$
\Delta F=\Delta F_{0}+\kappa_{2} \int_{0}^{z} d s \mathcal{E}\left(\theta_{0}, s\right) e^{i \theta_{0}} \frac{\partial}{\partial \eta} F_{0}\left(\theta_{0}, \eta, s\right),
$$

where $\theta_{0}=\theta-2 k_{u} \eta z+2 k_{u} \eta s$. Since the FEL gain becomes negligible when the width of $V(\eta)$ is much larger than the FEL parameter $\rho[1]$, where

$$
\rho=\left(\frac{e^{2} c Z_{0} K^{2} n_{0}}{32 \gamma_{0}^{3} m c^{2} k_{u}^{2} \sigma}\right)^{1 / 3},
$$

we have $2 k_{u} \eta z \sim 2 k_{u} \rho z \sim 2 \pi$ in the exponential gain regime. We can therefore make the approximation $\theta_{0} \approx \theta$ in the slowly varying $\mathcal{E}$ and $F_{0}$ but keep the fast oscillatory phase $e^{i \theta_{0}}$. Inserting Eqs. (16) and (17) into Eq. (14) and applying the Laplace transformation, we obtain

$$
\begin{aligned}
\mathcal{E}= & \frac{\kappa_{1} k_{f}}{k_{u}} \sum_{j}^{\theta_{j}<\theta} e^{-i \theta_{j}} \int \frac{d \lambda}{2 \pi i} e^{2 i \lambda\left[k_{u} z-\left(\theta-\theta_{j}\right)\right]} \int d \eta \frac{V(\eta)}{\lambda-\eta} \\
& \times \exp \left(-2 i \rho^{3} \int d \eta \frac{d V / d \eta}{\lambda-\eta} \int_{\theta_{j}}^{\theta} \chi\left(\theta^{\prime}\right) d \theta^{\prime}\right)
\end{aligned}
$$

The $\lambda$-integration is along a straight path parallel to the real axis and below all singularities of the integrand. It is nonzero only when $\theta-\theta_{j}<k_{u} z$ or $\xi-\xi_{j}<\xi_{s}$ (the slippage length). Hence the total electric field at $\theta$ is the sum of fields that originated from the discrete radiators prior to $\theta$ but within the slippage length. The electron gain medium is treated as a continuous fluid à la Vlasov and is justified in Ref. [4]. For a monochromatic beam with $V(\eta)=\delta(\eta)$, Eq. (19) reproduces the result of Ref. [2]. Coherent SASE can be evaluated by turning the sum into an integral following Eq. (5) and calculating the contribution of the essential singularity at $\lambda=0$ numerically [4]. 
Following Ref. [4], one can re-express Eq. (19) as:

$$
\begin{aligned}
\mathcal{E}(z, \theta)= & \frac{\kappa_{1} k_{f}}{2 k_{u}} \sum_{j}^{\theta_{j}<\theta} e^{-i \theta_{j}} \int_{-\infty+i \epsilon}^{\infty+i \epsilon} \frac{d \nu}{2 \pi i} e^{-i \nu\left(\theta-\theta_{j}\right)} \\
& \times \int \frac{d \lambda}{2 \pi i} \frac{e^{2 i \lambda k_{u} z}}{D\left(\lambda, \nu, \theta, \theta_{j}\right)} \int d \eta \frac{V(\eta)}{\lambda-\eta}
\end{aligned}
$$

where $\epsilon$ is an infinitesimal and positive number,

$$
D\left(\lambda, \nu, \theta, \theta_{j}\right)=\lambda-\frac{\nu}{2}+\rho^{3} w\left(\theta, \theta_{j}\right) \int d \eta \frac{d V / d \eta}{\lambda-\eta},
$$

and

$$
w\left(\theta, \theta_{j}\right)=\frac{1}{\left(\theta-\theta_{j}\right)} \int_{\theta_{j}}^{\theta} \chi\left(\theta^{\prime}\right) d \theta^{\prime} \leq 1 .
$$

For the coasting beam, $w\left(\theta, \theta^{\prime}\right)=1$ and $D(\lambda, \nu)=0$ is the dispersion relation including the energy spread [1]. Equation (21) provides a generalization to the bunched beam. When the bunch distribution does not change appreciably over the slippage length, $w\left(\theta, \theta_{j}\right) \approx \chi(\theta)$ from Eq. (22), and the FEL gain is affected only by the local electron current as expected.

\section{SIMULATION ALGORITHM}

In order to handle a general beam distribution and to study the nonlinear regime, we have developed a simulation code that is based on the individual particle formulation of FEL equations:

$$
\begin{aligned}
\frac{\partial \theta_{j}}{\partial \bar{z}} & =\bar{\eta}_{j}, \\
\frac{\partial \bar{\eta}_{j}}{\partial \bar{z}} & =-a e^{i \theta}+\text { c.c. }, \\
{\left[\frac{\partial}{\partial \bar{z}}+\frac{1}{2 \rho} \frac{\partial}{\partial \theta}\right] a } & =\chi(\theta)\left[e^{-i \theta}+\left\langle e^{-i \theta_{j}}\right\rangle\right],
\end{aligned}
$$

where $\bar{z}=2 k_{u} \rho z, \bar{\eta}=\eta / \rho$, and $a=2 \rho_{\theta} k_{u} \mathcal{E} /\left(\kappa_{1} n_{o}\right)$ is the scaled electric field. Equation (25) follows directly from Eq. (14), where the contribution of the smooth (coherent) distribution and the fluctuating (incoherent) part are explicitly separated into two terms. The partial derivative with respect to $\theta$ in Eq. (25) describes the slippage between the electron and the radiation field.

A time-dependent simulation algorithm [6] can be constructed to take into account the slippage effect: one first divides the bunch into $N_{b}=l_{b} / \lambda_{r}$ buckets and loads each bucket with simulation particles that are uniform in $\theta$ and have the proper energy spread. Apply Eqs. (23), (24) and (25) without the slippage term in each bucket, and then slip the computed field one bucket forward after each undulator period. To start up the FEL process, one either gives a small initial bunching $b_{0}[6]$ or uses the shot noise simulation algorithm of Ref. [7]. However, such a discretization is not adequate for CSE simulation because the bunch distribution function $\chi(\theta)$ is only sampled with a sampling

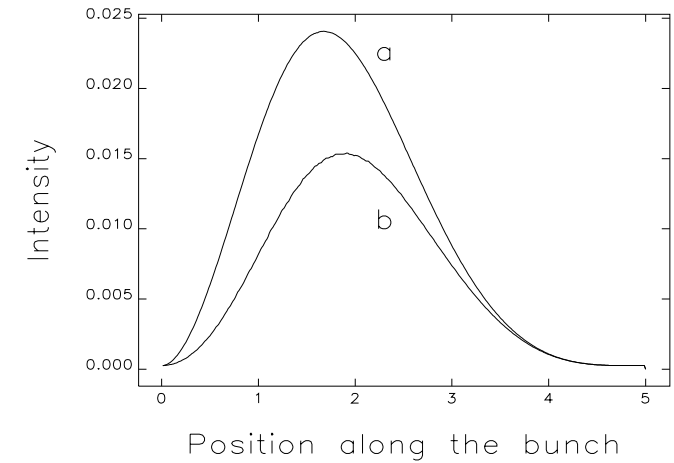

Figure 1: Coherent SASE intensity $\left|a_{c}\right|^{2}$ versus $2 \rho \theta$ ( $\rho=$ $1 /(40 \pi), \bar{z}=5$ ): (a) without initial energy spread, (b) with a flat-top energy spread of width $\rho$.

interval $\lambda_{r}$. Thus, the Fourier transform of $\chi(\theta)$ is defined only between the Nyquist critical frequency $f_{c}=c /\left(2 \lambda_{r}\right)$ or $\omega_{c}=c k_{f} / 2$, and the coherent bunching around the resonant frequency $c k_{f}$ is left out.

We modify this time-dependent approach to include the CSE effect by decreasing the sampling interval to cover the resonant part of the bunch spectrum. For example, we can divide the bunch into $8 N_{b}$ sections so that the critical frequency is $4 c k_{f}$. The spectral power outside this frequency range should be sufficiently small to eliminate the effect of aliasing. The electric field is computed and averaged over the resonant wavelength, in consistent with the slowly varying envelope approximation. Compared with the multi-frequency approach to CSE simulation [8], the time-dependent approach is more straightforward and can include the shot noise in a natural way. Figure 1 shows an example of simulation where we have intentionally turned the noise off. The bunch is assumed to be longer than the slippage length. CSE from the trailing part of the bunch within the slippage length (i.e., $0<2 \rho \theta<\bar{z}$ ) has been amplified and is shown in Fig. 1(a) for a case without initial energy spread and in Fig. 1(b) for the case with a flattop energy spread of width $\rho$. Figure 1(a) agrees very well with the calculation of Eq. (19). Such a simulation technique is also capable of studying the nonlinear behavior of the incoherent and the coherent SASE.

\section{REFERENCES}

[1] K. -J. Kim, Nucl. Instr. Meth. A 250, 396 (1986).

[2] J. -M. Wang and L. -H. Yu, Nucl. Instr. Meth. A 250, 484 (1986).

[3] K. -J. Kim and S. J. Hahn, Nucl. Instr. Meth. A 358, 93 (1995).

[4] S. Krinsky, Phys. Rev. E 59, 1171 (1999).

[5] R. Bonifacio, Opt. Comm. 138, 99 (1997).

[6] R. Bonifacio et al., Phys. Rev. A 40, 4467 (1989).

[7] C. Penman and B. W. J. McNeil, Opt. Comm. 90, 82 (1992).

[8] N. Piovella, AIP Conference Proceedings 413, 205 (1997). 